\title{
A Predictive Power Control Scheme for Energy Efficient State Estimation via Wireless Sensor Networks
}

\author{
Daniel E. Quevedo, Member, IEEE, and Anders Ahlén, Senior Member, IEEE
}

\begin{abstract}
We investigate state estimation via wireless sensor networks over fading channels causing random packet loss. Packet loss probabilities depend upon the time-varying channel gains and transmission power levels used by the sensors. We develop a predictive controller which trades off sensor energy expenditure versus state estimation accuracy. The latter is measured by the expected value of the future covariance matrices provided by the associated time-varying Kalman Filter. To further conserve energy at the sensors, the controller is located at the gateway and sends coarsely quantized power increment commands, only whenever necessary. Simulations based on real channel measurements show that the proposed approach gives excellent results.
\end{abstract}

\section{INTRODUCTION}

The interest in estimation and control over lossy communication links has increased tremendously in recent years; see, e.g., [1]-[3] and the references therein. In particular, with the rapid evolution of Wireless Sensor Networks (WSNs), see e.g., [4]-[7] for recent achievements, the use of wireless sensors (and actuators) has become an interesting alternative. The driving force behind this evolution from wired to wireless is the low deployment cost: There is no need for extensive wiring, either in new installations or for upgrading old systems. In addition, wireless sensors and actuators can be placed where wires cannot go, or where power sockets are not available.

A drawback of using wireless channels lies in that they are subject to fading and interference, which frequently lead to packet errors. ${ }^{1}$ Interestingly, the time-variability of the fading channel can be compensated for by adjusting the power levels. Thus, an important challenge is to design suitable power control schemes.

Depending on the application, the wireless channel can be constant (the environment is static and receiver and transmitter are not moving) or time varying. The time variability may be caused by moving objects, vehicles, people and so forth. Also, the receiver or the transmitter can be mounted on a moving object, which may be the case in process industry. Therefore, in addition to the propagation path loss, the channel may be subject to slow and/or fast fading.

Daniel Quevedo is with the School of Electrical Engineering \& Computer Science, The University of Newcastle, NSW 2308, Australia; e-mail: dquevedo@ieee.org.

Anders Ahlén is with the Department of Engineering Sciences, Signals and Systems, Uppsala University, PO Box 534 SE-751 21, Uppsala, Sweden; e-mail: Anders.Ahlen@signal.uu.se

${ }^{1}$ The situation is different to that of wired channels, where packet errors and delays are caused by congestion.
Beside the fading channel, another important issue, which arises in the absence of power sockets, is the need for energy conservation. Even though power scavenging is presently a hot topic in WSN research, saving energy is of utmost importance to avoid unnecessary maintenance, such as the replacement of batteries. Energy conservation has so far been given little attention in the literature, although some contributions have considered energy issues recently; see, e.g., [8]-[11]. In particular, [9] uses convex optimization to derive optimal power scheduling for a time-invariant decentralized case.

In this paper we will study optimal state estimation via WSNs when sensor data are transmitted over a fading channel generating random packet loss. The packet loss probabilities depend, in a nonlinear fashion, upon the time-varying channel gains and power levels used by the sensors. Thus, the packet loss process can be regarded as a disturbance, which, to some extent, can be manipulated by choosing transmission power levels. Since at the gateway side, past and present transmission outcomes are known, a time-varying Kalman Filter can be used. We will develop a centralized dynamic controller which is located at the gateway and decides upon the transmission power levels to be used by each sensor. The controller uses elements of predictive control [12] to trade off estimation accuracy against energy use.

To further conserve energy, the proposed controller sends coarsely quantized power increment commands to the sensors, only whenever necessary. Consequently, the system to be controlled is not only nonlinear and stochastic (due to the occurrence of random packet errors), but also subject to finite-set constraints on decision variables. Key to keeping the computational burden limited is the fact that the occurrence of packet errors constitutes a binary random variable, thus, expected values can be exactly evaluated via finite sums over the possible transmission outcome scenarios, i.e., no integrals need to be evaluated or approximated.

Before proceeding, we emphasize that in our approach, the sensors are commanded by the controller to wake up, collect data, transmit the data at the desired power level, and go to sleep. This would keep processing power at the sensors to a minimum. Scheduling schemes for related problems have been proposed recently in the literature. For example, in [13] a sensor selection schedule is presented which minimizes a steady state estimation error covariance. In that work, sensors share state information over error-free links and only one sensor is allowed to transmit at a time. Here, we consider time-varying channels affected by errors and propose a power 
controller, which jointly minimizes the state estimation error covariance and transmission power levels.

The remainder of this paper is organized as follows: In Section II, the state estimation problem is formulated. Section III describes energy aspects. Section IV presents the predictive power controller which trades off state estimation error versus energy usage and respects constraints on power levels and their increments. Simulation results with channel measurements are documented in Section V. Finally, in Section VI conclusions are given.

\section{State Estimation via Sensor Networks}

Consider an uncontrolled LTI $n$-dimensional system:

$$
x(k+1)=A x(k)+w(k), \quad k \in \mathbb{N}_{0} \triangleq\{0,1, \ldots\},
$$

where the initial system state is Gaussian distributed with mean $x_{0}$ and covariance $P_{0} \in \mathbb{R}^{n \times n}$, i.e., $x(0) \in \mathcal{N}\left(x_{0}, P_{0}\right)$. Similarly, the driving noise process $w=\{w(k)\}_{k \in N_{0}}$ is i.i.d., where each $w(k) \in \mathcal{N}(0, Q)$.

To remotely estimate the system state sequence $x=$ $\{x(k)\}_{k \in N_{0}}$, a network of $M$ sensors is used. Each sensor provides a scalar noisy measurement signal, say $y_{m}=$ $\left\{y_{m}(k)\right\}_{k \in N_{0}}$ :

$$
y_{m}(k)=C_{m} x(k)+v_{m}(k), \quad m \in\{1,2, \ldots, M\},
$$

where $v_{m}=\left\{v_{m}(k)\right\}_{k \in N_{0}}$ is an i.i.d. process with each $v_{m}(k) \in \mathcal{N}\left(0, R_{m}\right) .^{2}$

The $M$ values in (2) are transmitted through wireless links to the single gateway. The received signals are then used to remotely estimate the state of the system (1).

Since the $M$ links between sensors and gateway are wireless, transmission errors are likely to occur. ${ }^{3}$ Faulty packets will be discarded when estimating the system state. We will model transmission effects by introducing the $M$ binary stochastic arrival processes $\gamma_{m}=\left\{\gamma_{m}(k)\right\}_{k \in N_{0}}$, $m \in\{1,2, \ldots, M\}$, where:

$\gamma_{m}(k)= \begin{cases}1 & \text { if } y_{m}(k) \text { arrives error-free at time } k, \\ 0 & \text { if } y_{m}(k) \text { does not arrive error-free at time } k .\end{cases}$

For further reference, we also define the overall arrival process of all $M$ links, say $\gamma=\{\gamma(k)\}_{k \in N_{0}}$, via:

$$
\gamma(k) \triangleq\left[\begin{array}{llll}
\gamma_{1}(k) & \gamma_{2}(k) & \ldots & \gamma_{M}(k)
\end{array}\right]^{T}, \quad k \in \mathbb{N}_{0} .
$$

In the present work, we will assume that the data transmitted incorporates error detection coding [15]. Hence, the gateway knows, whether packets received from the sensors contain errors or not. Thus, at any time $k$, past and present realizations of the overall transmission process, say $\gamma^{k}$, are available at the gateway.

For state estimation purposes, the system amounts to sampling (1)-(2) only at the successful transmission instants

\footnotetext{
${ }^{2}$ In addition to measurement noise, $v_{m}$ may also describe quantization effects, which we model as white and Gaussian.

${ }^{3}$ In the present work, we will assume that sensor data is not affected by delays or Multiple Access Interference (MAI). Extensions of our framework to include MAI and time-delay issues, and also irregular sampling [14], does not present any conceptual difficulties.
}

of each sensor link. Indeed, the conditional probability distribution of the system state at any time $k$, given $\gamma^{k}$ and correctly received sensor measurements up to time $k$, say $y^{k}$, is Gaussian. Consequently, the conditional mean of $x(k)$ and covariance of the state estimation error, i.e,

$$
\begin{aligned}
& \hat{x}(k) \triangleq \mathcal{E}_{w, v, x(0)}\left\{x(k) \mid y^{k}, \gamma^{k}\right\} \\
& \bar{P}(k) \triangleq \mathcal{E}_{w, v, x(0)}\left\{(\hat{x}(k)-x(k))(\hat{x}(k)-x(k))^{T} \mid y^{k}, \gamma^{k}\right\},
\end{aligned}
$$

satisfy the Kalman Filter recursions (see, e.g., [16]): ${ }^{4}$

$$
\begin{aligned}
\hat{x}(k+1) & =A \hat{x}(k)+K(k+1)(y(k+1)-C(k+1) A \hat{x}(k)) \\
P(k+1) & =A P(k) A^{T}+Q-A K(k) C(k) P(k) A^{T} \\
\bar{P}(k) & =P(k)-K(k) C(k) P(k),
\end{aligned}
$$

where:

$$
\begin{aligned}
C(k) & \triangleq\left[\begin{array}{lll}
\gamma_{1}(k)\left(C_{1}\right)^{T} \quad \gamma_{2}(k)\left(C_{2}\right)^{T} \quad \ldots \quad \gamma_{M}(k)\left(C_{M}\right)^{T}
\end{array}\right]^{T} \\
K(k) & \triangleq P(k) C(k)^{T}\left(C(k) P(k) C(k)^{T}+R\right)^{-1} \\
R & \triangleq \operatorname{diag}\left(R_{1}, R_{2}, \ldots, R_{M}\right)
\end{aligned}
$$

and with initial values $P(0)=P_{0}$ and $\hat{x}(0)=x_{0}$.

In the following section we will show how the arrival process $\gamma$ in (3) depends upon the transmission power used by the sensors. In Section IV, we will then use the time-varying Kalman filter presented above, to develop a predictive power controller for the sensor network state estimation problem.

\section{Power Issues in Wireless Sensor Networks}

In this section we will discuss some fundamental aspects on power control for wireless sensor networks.

As mentioned in Section II, for each of the $M$ channels, $\gamma_{m}(k)$ is a Bernoulli trial with success probability

$$
\lambda_{m}(k) \triangleq \mathcal{P}\left\{\gamma_{m}(k)=1\right\} .
$$

This probability is, in general, time-varying and depends upon the propagation environment and on the transmission power used by the sensor radio power amplifiers. Indeed, in general it holds that:

$$
\lambda_{m}(k)=f_{m}\left(u_{m}(k) h_{m}(k)\right), \quad m \in\{1,2, \ldots, M\},
$$

where $f_{m}(\cdot):[0, \infty) \rightarrow[0,1]$ is a monotonically increasing function, which depends upon the modulation scheme employed.

In (6), $h_{m}(k)$ denotes the square of the magnitude of the channel gain. (Note that $h_{m}(k)$ is here defined to include also path-loss, power amplifier efficiency, antenna gain and noise figure.) The term $u_{m}(k)$ denotes the power used by the $m$-th radio power amplifier.

It follows from the reasoning above, that one can improve transmission reliability and, thus, state estimation accuracy for a given wireless propagation environment, by simply increasing the power used by the transmitter. However, in wireless sensor networks, it is of fundamental importance to save energy: Sensor nodes are expected to be operational for

\footnotetext{
${ }^{4}$ The one sensor case, i.e., where $M=1$, was investigated in [17].
} 
several years without maintenance. This motivates us to use the available energy resources with care.

We will quantify the energy used by each sensor $m \in$ $\{1, \ldots, M\}$ at a given (discrete) time instant via $g_{m}\left(u_{m}(k)\right)$, where

$$
g_{m}(\cdot):[0, \infty) \rightarrow[0, \infty)
$$

is a monotonically increasing function. The total energy used by the $M$ sensors at each time instant $k$ is, thus, given by:

$$
G(u(k)) \triangleq \sum_{m=1}^{M} g_{m}\left(u_{m}(k)\right),
$$

where

$$
u(k) \triangleq\left[\begin{array}{llll}
u_{1}(k) & u_{2}(k) & \ldots & u_{M}(k)
\end{array}\right]^{T} .
$$

We note that the energy spent by the sensor radio power amplifier for transmitting each measurement value is $b u_{m}(k) / r$ where $r$ is the channel bit rate used and $b$ is the number of bits per measurement value. ${ }^{5}$

In order to save energy required to process the received signal at the sensors, we would like to keep the signaling from the gateway to the sensors as low as possible. This motivates us to send only a few bits indicating whether power should be kept as is, be increased, or decreased. The available transmission power will also be constrained in magnitude. Thus,

$$
0 \leq u_{m}(k) \leq u_{m}^{\max }, \quad \forall k \in \mathbb{N}_{0}, \forall m \in\{1,2, \ldots, M\},
$$

for given values $\left\{u_{m}^{\max }\right\}$.

We conclude from the above that power control design involves a trade-off between transmission error probabilities (and, thus, state estimation accuracy) and energy consumption.

Remark 1 (Channel Gain Predictors): We note from (6) that power control ideally would require present and future channel gains. These values can be estimated by transmitting a fixed number of pilot bits based on which the channel can be estimated. These and previously estimated channel gains may then also be used for predicting future channel gains, see, e.g., [18], [19]. Note that, even if received packets are discarded for state estimation, they may still be used for channel gain estimation and prediction.

Example 1 (Constant AWGN Channel and BPSK): If Binary Phase Shift Keying is used over an additive white Gaussian noise channel with constant signal-to-noise ratio, say SNR, then the Bit Error Rate (BER), here denoted $B$, is given by [15]

$$
B=\bar{Q}(\sqrt{2 \mathrm{SNR}}),
$$

where $\bar{Q}(\cdot)$ is the Q-function defined by

$$
\bar{Q}(z) \triangleq \frac{1}{\sqrt{2 \pi}} \int_{z}^{\infty} e^{-\eta^{2} / 2} d \eta
$$

\footnotetext{
${ }^{5}$ In addition to the energy used by the radio power amplifier, there are also energy costs associated with the transmission and reception of each bit. These costs are caused by the circuitry involved, e.g., DA-converters, mixers, and oscillators. Furthermore, one may also include the cost for wake-up and sensing. We will, however, not consider these aspects in more detail here.
}

Although the above model is only valid in the timeinvariant case, we shall adopt it also for time-varying channels and power levels. For that purpose, we introduce the instantaneous signal-to-noise ratio for each channel $m$ via $^{6}$

$$
\operatorname{SNR}_{m}(k)=\frac{h_{m}(k) u_{m}(k)}{r k_{B} T},
$$

where $k_{B}$ is the Boltzmann constant (in Joule per Kelvin) and $T$ is the temperature (in Kelvin).

To characterize the effect of packet length, let each measurement value, to be transmitted over the fading channel, consist of $b$ bits, including error detection bits. If we adopt a block fading model, where the channel is constant over the duration of one packet, but may be subject to fading between packets, then the probability of successful packet arrival (5) is simply given by ${ }^{7}$

$$
\lambda_{m}(k)=\left(1-B_{m}(k)\right)^{b} .
$$

In this light, the functions $f_{m}(\cdot)$ and $g_{m}(\cdot)$ in (6) and (7), respectively, are now readily found to be:

$$
\begin{aligned}
f_{m}\left(u_{m}(k) h_{m}(k)\right) & =\left(1-\bar{Q}\left(\sqrt{\frac{2 h_{m}(k) u_{m}(k)}{r k_{B} T}}\right)\right)^{b} \\
g_{m}\left(u_{m}(k)\right) & =(b / r) u_{m}(k) .
\end{aligned}
$$

We will use this model in Section V.

\section{Predictive Power Control}

In what follows, we will present a predictive control strategy which minimizes a cost function, which takes into account energy consumption and estimation quality over a future prediction horizon. To keep processing at the sensors to a minimum, the controller is located at the gateway and its output is coarsely quantized, i.e., each value has a short word-length.

\section{A. Control Signal Coding}

Since we are tackling energy issues, in the present work we choose to use only a small number of bits for the power control commands sent from the gateway to the sensors. This incurs only limited reception and processing power at the sensor sides. Here, we will use coding ideas frequently used in power control architectures for cellular networks, see, e.g., [20] (and compare also to our work on Networked Control Systems in [21]). We, thus, send coarsely quantized power increments, say $\Delta u_{m}(k)$, rather than actual power values, $u_{m}(k)$, to each sensor $m \in\{1,2, \ldots, M\}$. All signals $\left\{\Delta u_{m}\right\}$ are constrained according to:

$$
\Delta u_{m}(k) \in \mathbb{U}_{m}, \quad \forall k \in \mathbb{N}_{0}, \forall m \in\{1,2, \ldots, M\},
$$

where $\left\{\mathbb{U}_{m}\right\}$ are given finite sets, each having a small number of elements.

\footnotetext{
${ }^{6} \mathrm{SNR}_{m}(k)$ denotes the signal-to-noise ratio at the receiver, as seen by the coherent detector.

${ }^{7}$ The instantaneous BER $B_{m}(k)$ is obtained by inserting $\operatorname{SNR}_{m}(k)$ into (10). Note that if the received packet is found to have at least one bit error, then it is discarded. The discarded packet may however still be
} used for channel estimation, see Remark 1. 
In our approach, upon receipt of $\Delta u_{m}(k)$, each sensor $m$ reconstructs the power level to be used by its radio power amplifier by simply setting

$$
u_{m}(k)=u_{m}(k-1)+\Delta u_{m}(k) .
$$

For further reference, we define the signal:

$$
\Delta u(k) \triangleq\left[\begin{array}{lll}
\Delta u_{1}(k) & \ldots & \Delta u_{M}(k)
\end{array}\right]^{T}, \quad k \in \mathbb{N}_{0} .
$$

Note that the quantization constraint on the transmitted values, see (12), imposes

$$
\Delta u(k) \in \mathbb{U} \triangleq \mathbb{U}_{1} \times \mathbb{U}_{2} \times \cdots \times \mathbb{U}_{M}, \quad \forall k \in \mathbb{N}_{0} .
$$

\section{B. Predictive Power Controller}

At each time instant $k \in \mathbb{N}_{0}$, the predictive power controller calculates the value of the current posterior covariance matrix $\bar{P}(k)$, which results from iterating (4) for the (known) past arrival process realizations $\gamma^{k}$, see (3), and uses channel gain predictions over a finite horizon of fixed length $N$ (recall Remark 1), namely:

$$
\left\{\hat{h}_{m}(k+1 \mid k), \ldots, \hat{h}_{m}(k+N \mid k)\right\}, \quad \forall m \in\{1,2, \ldots, M\} .
$$

With this information, the controller minimizes the finite-set constrained cost function

$$
J(\Delta U) \triangleq \mathcal{E}_{\Gamma(k)}\left\{J_{1}(\Gamma(k), \Delta U)\right\}+\rho J_{2}(\Delta U),
$$

where $\rho \geq 0$ is a design parameter which allows one to trade estimation accuracy for energy consumption.

The stochastic aspect of the power control problem, namely the possibility of transmission errors, is captured in (16) by the discrete random variable

$$
\Gamma(k) \triangleq\left[\begin{array}{llll}
\gamma(k+1) & \gamma(k+2) & \ldots & \gamma(k+N)
\end{array}\right]^{T} .
$$

Accordingly, $\mathcal{E}_{\Gamma(k)}$ denotes expectation taken with respect to the probability mass distribution of $\Gamma(k)$. Note that this distribution depends upon the power levels and channel gains, see (5) and (6). Thus, $\Gamma(k)$ can be regarded as a controlled stochastic disturbance to the system.

In (16), the estimation error covariance and energy use are quantified via

$$
\begin{aligned}
J_{1}(\Gamma(k), \Delta U) & \triangleq \sum_{\ell=k+1}^{k+N} \operatorname{trace}\left(\bar{P}^{\prime}(\ell)\right) \\
J_{2}(\Delta U) & \triangleq \sum_{\ell=k+1}^{k+N} G\left(u^{\prime}(\ell)\right),
\end{aligned}
$$

respectively. The decision variables, i.e, the vector of tentative future power value increments, are collected in ${ }^{8}$

$$
\Delta U=\left\{\Delta u^{\prime}(k+1), \Delta u^{\prime}(k+2), \ldots, \Delta u^{\prime}(k+N)\right\}
$$

see (13). In accordance with (8) and (14), $\Delta U$ yields the tentative future power levels $u^{\prime}(\ell)$ in (17) via

$$
u^{\prime}(\ell)=u^{\prime}(\ell-1)+\Delta u^{\prime}(\ell), \quad \ell \in\{k+1, \ldots, k+N\},
$$

\footnotetext{
${ }^{8}$ In the sequel, primed variables refer to tentative values of the corresponding physical variables.
}

starting from the current power levels, i.e., $u^{\prime}(k)=u(k)$.

Finally, trace $\left(\bar{P}^{\prime}(\ell)\right)$ denotes the trace of the a posteriori conditional state covariance matrix obtained from iterating (4) with initial value $P(k+1)$ and for a given realization of $\Gamma(k)$.

At each time instant $k \in \mathbb{N}_{0}$, and given channel gain predictions in (15), the controller finds the optimizing sequence

$$
\Delta U^{\mathrm{opt}} \triangleq \arg \min J(\Delta U),
$$

subject to the constraints:

$$
\begin{array}{ll} 
& \Delta U \in \mathbb{U}^{N} \\
0 u_{m}^{\prime}(\ell) \leq u_{m}^{\max }, & \forall \ell \in\{k+1, \ldots, k+N\}, \forall m,
\end{array}
$$

where

$$
\mathbb{U}^{N} \triangleq \mathbb{U} \times \mathbb{U} \times \cdots \times \mathbb{U} .
$$

Following the moving horizon principle, see, e.g., [12], at each time $k$, the proposed controller sends only the $M$ power updates contained in ${ }^{9}$

$$
\Delta u(k+1)^{\mathrm{opt}} \triangleq\left[\begin{array}{llll}
I_{M} & 0_{M} & \ldots & 0_{M}
\end{array}\right] \Delta U^{\mathrm{opt}}
$$

to the corresponding sensors. At the next time instant, namely $k+1$, the optimization procedure is repeated, giving rise to control increments $\Delta u(k+2)^{\text {opt }}$. This procedure is repeated ad infinitum.

The proposed power controller jointly decides upon the power levels of all $M$ sensors by using future channel gain predictions. The control law respects finite word-length constraints and takes into account the coding system used for the power level signaling, see Section IV-A. We emphasize that, for general time-varying channels, the power levels obtained are not constant, but are assigned dynamically through optimization of the criterion in (16) and, thus, optimize the resulting performance.

\section{Computational Aspects}

We note that minimization of $J(\Delta U)$ in (19) is carried out on-line at the gateway, where computational aspects play less of a role than at the sensors. Nevertheless, in what follows, we will briefly outline some computational aspects.

Despite the fact that we are dealing with a stochastic nonlinear optimization problem, solving (19) in real-time is surprisingly simple, due to the finite-set nature of $\Gamma(k)$ and of $\Delta U$. To be more precise, $\Gamma(k)$ takes only values in the finite set $\{0,1\}^{M N}$, whose elements we will denote via $d_{i}$, where $i \in\left\{0,1, \ldots, 2^{M N}-1\right\}$. The probability of a given arrival process realization occurring, namely

$$
\mathcal{P}_{i}(k) \triangleq \mathcal{P}\left\{\Gamma(k)=d_{i}\right\}
$$

depends upon future channel gains and power levels used by the sensors. These probabilities can be calculated by simply forming the product of the individual terms

\footnotetext{
${ }^{9} I_{M}$ denotes the $M \times M$ identity matrix and $0_{M} \triangleq 0 \cdot I_{M}$.
} 
$f_{m}\left(u_{m}(\ell) h_{m}(\ell)\right)$, see (6). Consequently, taking expectation in (16) reduces to evaluating the finite sum:

$\mathcal{E}_{\Gamma(k)}\left\{J_{1}(\Gamma(k), \Delta U)\right\}=\sum_{i=0}^{2^{M N}-1} \mathcal{P}_{i}(k)\left(\sum_{\ell=k+1}^{k+N} \operatorname{trace}\left(\bar{P}^{\prime}(\ell)\right)\right)$

where the sum of the traces on the RHS is carried out for each realization $d_{i}$ and is independent of $\Delta U$.

Furthermore, the optimization (19) requires evaluating $J(\Delta U)$ for at $\operatorname{most}^{10}|\mathbb{U}|^{N}$ possibilities of $\Delta U$, see also [22]. Thus, the number of calculations to be carried out at each time instant is proportional to, at most, $\left(2^{M}|\mathbb{U}|\right)^{N} .{ }^{11}$ Note that, if $\Gamma(k)$ would be a continuous random variable and/or $\Delta U$ would not be finite-set constrained, then the optimization procedure would, in general, be significantly more involved.

\section{Simulation Study}

In this section we will evaluate the proposed power controller on measured channel data, which were acquired in an office space area at the Signals and Systems Group at Uppsala University, Sweden. The transmitter was placed in an office and the receiver was located $8 \mathrm{~m}$ away in the corridor outside the office. The transmitter position was fixed, whereas the receiver was mounted on a rail allowed to move over a distance of $1.25 \mathrm{~m}$, perpendicular to the corridor. Measurements were collected at the $2.4 \mathrm{GHz}$ ISM band. Fig. 1 illustrates normalized channel gains of two realizations, one with horizontal and one with vertical polarization. ${ }^{12}$

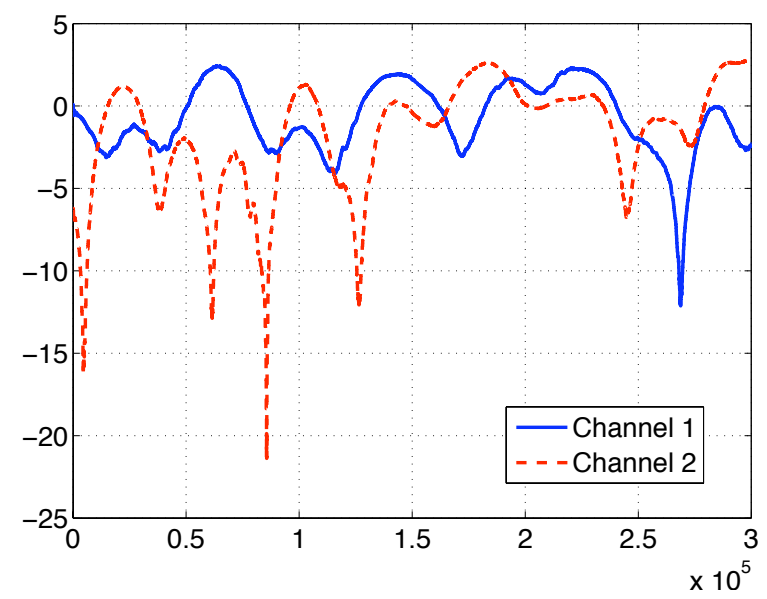

Fig. 1. Normalized channel magnitudes (in dB), $\sqrt{h_{1}}$ (solid) and $\sqrt{h_{2}}$ (dashed). The horizontal axis is scaled in samples, where $3 \cdot 10^{5}$ corresponds to approximately $1.25 \mathrm{~m}$.

Fig. 1 illustrates the channel gains as a function of relative position, and, thus, corresponds to what a receiver would perceive if it moved through space. The gains shown in Fig. 1

\footnotetext{
${ }^{10}|\mathbb{U}|$ denotes the cardinality of the finite set $\mathbb{U}$.

${ }^{11}$ Future work may include the development of computationally more attractive optimization algorithms.

${ }^{12}$ The channels were found to be well described by time-varying gains. It is worth emphasizing here that, since we have real channel data, in this case we do not require to ascribe probability distributions to the fading. However, if we had knowledge about underlying fading statistics, then it could easily be incorporated, e.g., by averaging (10) over the fading distribution.
}

also give an indication of what fading to expect, if objects are moving between fixed sensor and gateway localizations. We note that the channels vary considerably, with some dips dropping as much as $20 \mathrm{~dB}$. Without appropriate dynamic power control, such large variations would require excessive energy consumption to avoid frequent transmission errors, and thus degraded estimation quality.

We will now apply the predictive power controller proposed in Section IV with parameters $N=1$ and $\rho=$ $200(\mu W)^{-1}$ on the measured channel data depicted in Fig. 1. For that purpose, we will adopt the simple expressions (10)-(11) $)^{13}$ and consider a second order state space model (1) with $Q=I_{2}, R=(1 / 100) I_{2}$,

$$
A=\left[\begin{array}{cc}
1 & 0 \\
1 & 0.7
\end{array}\right], C_{1}=\left[\begin{array}{ll}
1 & 0
\end{array}\right], C_{2}=\left[\begin{array}{ll}
0 & 1
\end{array}\right] .
$$

The constraints on the power values, see (9), are

$$
u_{1}^{\max }=u_{2}^{\max }=40 \mu \mathrm{W} .
$$

Increments are restricted to belong to the finite sets

$$
\mathbb{U}_{1}=\mathbb{U}_{2}=\{0, \pm 3 \mu \mathrm{W}\} .
$$

We will furthermore assume that the controller has perfect one-step-ahead channel predictions. ${ }^{14}$

Fig. 2 illustrates that the controller tries to find the best compromise between the two sensors links. ${ }^{15}$ In particular, the controller at times approximately inverts the channel gains. At other times, it decides to send one of the sensors to sleep, i.e., to set $u_{1}(k)=0$ or $u_{2}(k)=0$.

To give further insight, Fig. 3 shows the success probabilities for both channels together with the achieved cost:

$$
V(k) \triangleq \operatorname{trace}(\bar{P}(k))+\rho G\left(u^{\prime}(k)\right) .
$$

Note that when the channel energies are small and, thus, $\lambda_{1}(k)$ and $\lambda_{2}(k)$ drop to very low levels, $V(k)$ quickly increases, since the Kalman filter becomes blind to the evolution of the states.

\section{CONCLUSiOnS}

An energy efficient power control scheme for state estimation via wireless sensor networks operating over fading channels was presented. The time variability of the fading channels frequently leads to transmission errors with subsequent random packet drops. State estimation in the face of intermittent observations was then performed by a timevarying Kalman filter.

In our scheme, the transmission power of the radio amplifier of each of the wireless sensors was controlled by the gateway. For that purpose, we proposed a predictive controller which utilizes predictions of radio channel gains and incorporates signal coding. Performance was illustrated

\footnotetext{
${ }^{13} \mathrm{We}$ choose $r k_{B} T=2.5 \cdot 10^{-10} \mu \mathrm{W}$, which corresponds to $r=60$ $\mathrm{kbits} / \mathrm{s}$ at room temperature. The number of bits per sensor value is 8 .

${ }^{14}$ Good accuracy can be obtained for prediction lengths up to a quarter of the carrier wavelength [18], [19].

${ }^{15}$ The mean values of $h_{1}(k)$ and $h_{2}(k)$ were $3.33 \cdot 10^{-11}$, i.e., $-105 \mathrm{~dB}$. At the maximum allowed power level, this corresponds to an SNR of $7 \mathrm{~dB}$.
} 

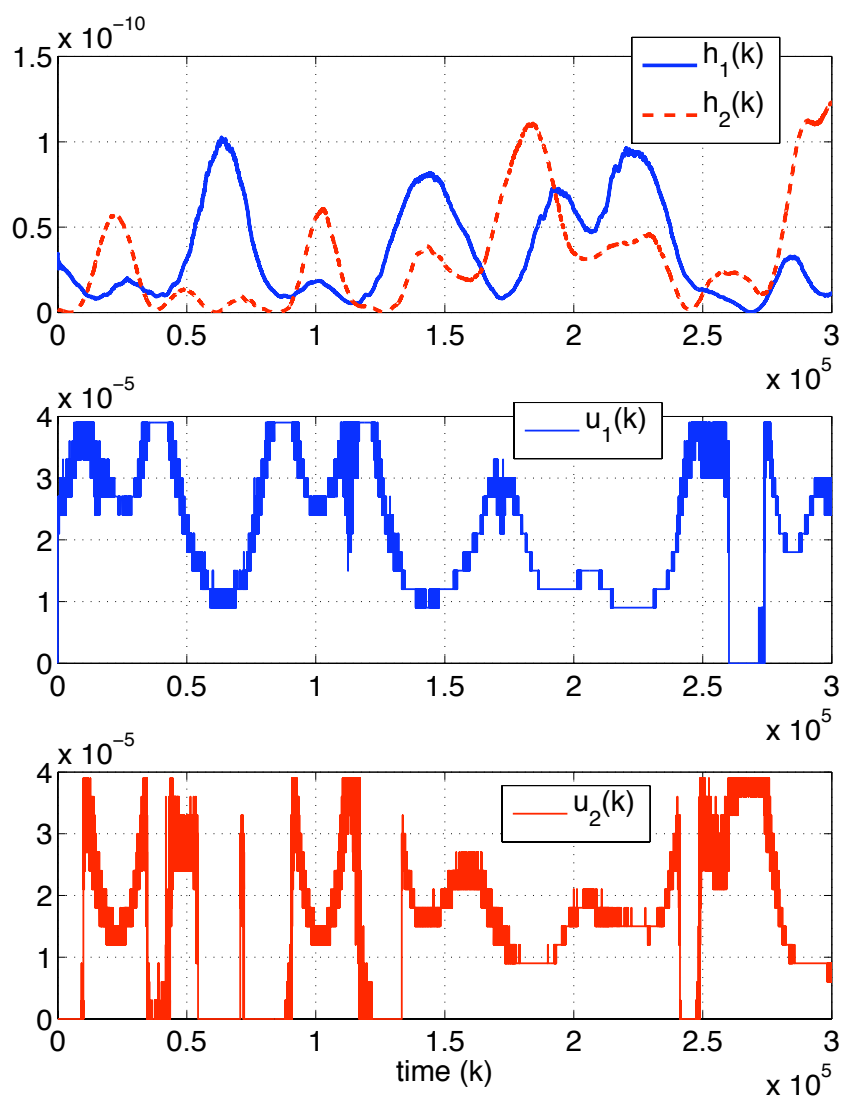

Fig. 2. Channel gains $h_{1}$ and $h_{2}$ (top) together with control signals $u_{1}(k)$ (middle) and $u_{2}(k)$ (bottom) provided by the predictive controller.

on measured channel data and the optimal control action was shown to find a suitable compromise between estimation quality and the amount of energy used.

\section{REFERENCES}

[1] J. P. Hespanha, P. Naghshtabrizi, and Y. Xu, "A survey of recent results in networked control systems," Proc. IEEE, vol. 1, pp. 138-162, Jan. 2007.

[2] G. N. Nair, F. Fagnani, S. Zampieri, and R. J. Evans, "Feedback control under data rate constraints: An overview," Proc. IEEE, vol. 95, pp. 108-137, Jan. 2007.

[3] L. Schenato, B. Sinopoli, M. Franceschetti, K. Poolla, and S. S. Sastry, "Foundations of control and estimation over lossy networks," Proc. IEEE, vol. 95, pp. 163-187, Jan. 2007.

[4] M. Ilyas, I. Mahgoub, and L. Kelly, Handbook of Sensor Networks: Compact Wireless and Wired Sensing Systems. Boca Raton, FL, USA: CRC-Press, Inc, 2004.

[5] X. Shen, Q. Zhang, and R. Caiming Qiu, "Wireless sensor networking [guest ed.]," IEEE Wireless Commun., vol. 14, pp. 4-5, Dec. 2007.

[6] P. Havinga, J. C. Hou, and F. Zhao, "Wireless sensor networks [guest ed.]," IEEE Wireless Commun., vol. 11, pp. 4-5, Dec. 2004.

[7] H. Gharavi and S. P. Kumar, "Special section on sensor networks and applications," Proc. IEEE, vol. 91, pp. 1151-1152, Aug. 2003.

[8] R. Wen, I. Mareels, and B. Krongold, "Optimal power analysis for network lifetime balance in hierarchy networks," in Proc. IFAC World Congr., (Seoul, Korea), 2008.

[9] J.-J. Xiao, S. Cui, Z.-Q. Luo, and A. J. Goldsmith, "Linear coherent decentralized estimation," IEEE Trans. Signal Processing, vol. 56, pp. 757-770, Feb. 2008.

[10] E. Björnemo, A. Ahlén, and M. Johansson, "On the energy-efficiency of cooperative MIMO in Nakagami fading wireless sensor networks," in Proc. 41st Asilomar Conf. Signals, Systems, and Computers, (Monterey, CA), 2007.
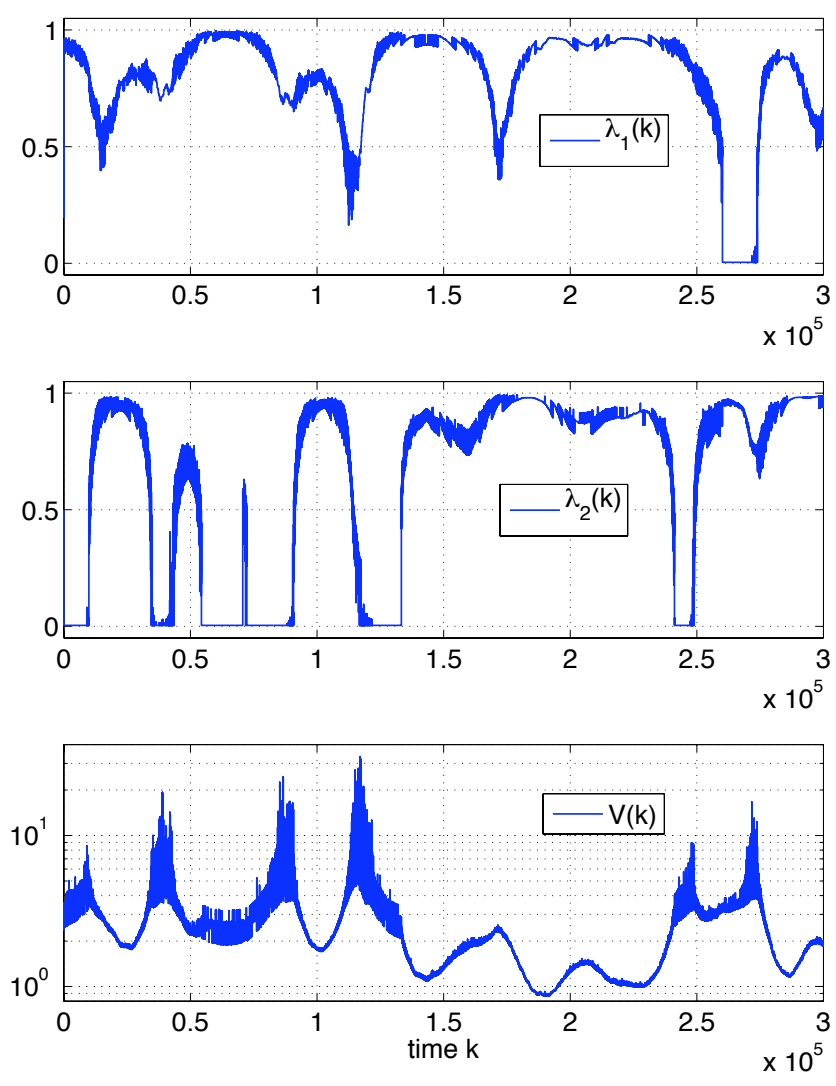

Fig. 3. Success Probabilities $\lambda_{m}(k)$ for Channel 1 and Channel 2, and the achieved cost $V(k)$ when the predictive power controller is employed.

[11] M. Johansson, E. Björnemo, and A. Ahlén, "Fixed link margins outperform power control in energy-limited wireless sensor networks," in Proc. IEEE Int. Conf. Acoust. Speech Signal Process., vol. 3, (Honolulu, HI), pp. 513-516, 2007.

[12] D. Q. Mayne, J. B. Rawlings, C. V. Rao, and P. O. M. Scokaert, "Constrained model predictive control: Optimality and stability," $\mathrm{Au}$ tomatica, vol. 36, pp. 789-814, June 2000.

[13] V. Gupta, T. H. Chung, B. Hassibi, and R. M. Murray, "On a stochastic sensor selection algorithm with applications in sensor scheduling and sensor coverage," Automatica, vol. 42, pp. 251-260, 2006.

[14] K. J. Åström and B. M. Bernhardsson, "Comparison of Riemann and Lebesgue sampling for first order stochastic systems," in Proc. IEEE Conf. Decis. Contr., pp. 2011-2016, 2002.

[15] J. G. Proakis, Digital Communications. New York, N.Y.: McGrawHill, 3rd ed., 1995.

[16] B. D. O. Anderson and J. Moore, Optimal Filtering. Englewood Cliffs, NJ: Prentice Hall, 1979.

[17] B. Sinopoli, L. Schenato, M. Franceschetti, K. Poolla, M. I. Jordan, and S. S. Sastry, "Kalman filtering with intermittent observations," IEEE Trans. Automat. Contr., vol. 49, pp. 1453-1464, Sept. 2004.

[18] T. Ekman, M. Sternad, and A. Ahlén, "Unbiased power prediction of Rayleigh fading channels," in Proc. Vehicular Technology Conf., vol. 1, pp. 280-284, 2002.

[19] T. Ekman, Prediction of Mobile Radio Channels: Modeling and Design. PhD thesis, Uppsala University, Oct. 2002.

[20] H. Holma and A. Toskala, eds., WCDMA for UMTS. West Sussex, England: John Wiley \& Sons, third ed., 2004.

[21] G. C. Goodwin, H. Haimovich, D. E. Quevedo, and J. S. Welsh, "A moving horizon approach to networked control system design," IEEE Trans. Automat. Contr., vol. 49, pp. 1427-1445, Sept. 2004.

[22] D. E. Quevedo, G. C. Goodwin, and J. A. De Doná, "Finite constraint set receding horizon quadratic control," Int. J. Robust Nonlin. Contr., vol. 14, pp. 355-377, Mar. 2004. 